\title{
Beta Radiation Enhanced Thermionic Emission from Diamond Thin Films
}

\author{
Alex Croot', Gary Wan', Alex Rowan², Hugo D. Andrade', James A. Smith ${ }^{2}$ \\ and Neil A. Fox ${ }^{1,2 *}$ \\ 'School of Physics, HH Wills Physics Laboratory, University of Bristol, Bristol, United Kingdom, ${ }^{2}$ School of Chemistry, \\ Cantock's Close, University of Bristol, Bristol, United Kingdom
}

OPEN ACCESS

Edited by:

David B. Go,

University of Notre Dame,

United States

Reviewed by:

Terence Musho,

West Virginia University,

United States

Patrick Jacob Shamberger,

Texas A\&M University,

United States

${ }^{*}$ Correspondence:

Neil A. Fox

neil.fox@bristol.ac.uk

Specialty section:

This article was submitted to Thermal and Mass Transport,

a section of the journal

Frontiers in Mechanical Engineering

Received: 31 July 2017

Accepted: 24 October 2017

Published: 13 November 2017

Citation:

Croot A, Wan G, Rowan A, Andrade HD, Smith JA and Fox NA (2017) Beta Radiation Enhanced

Thermionic Emission from

Diamond Thin Films.

Front. Mech. Eng. 3:17. doi: 10.3389/fmech.2017.00017
Diamond-based thermionic emission devices could provide a means to produce clean and renewable energy through direct heat-to-electrical energy conversion. Hindering progress of the technology are the thermionic output current and threshold temperature of the emitter cathode. In this report, we study the effects on thermionic emission caused by in situ exposure of the diamond cathode to beta radiation. Nitrogen-doped diamond thin films were grown by microwave plasma chemical vapor deposition on molybdenum substrates. The hydrogen-terminated nanocrystalline diamond was studied using a vacuum diode setup with a ${ }^{63} \mathrm{Ni}$ beta radiation source-embedded anode, which produced a 2.7-fold increase in emission current compared to a ${ }^{59} \mathrm{Ni}$-embedded control. The emission threshold temperature was also examined to further assess the enhancement of thermionic emission, with ${ }^{63} \mathrm{Ni}$ lowering the threshold temperature by an average of $58 \pm 11{ }^{\circ} \mathrm{C}$ compared to the ${ }^{59} \mathrm{Ni}$ control. Various mechanisms for the enhancement are discussed, with a satisfactory explanation remaining elusive. Nevertheless, one possibility is discussed involving excitation of preexisting conduction band electrons that may skew their energy distribution toward higher energies.

Keywords: thermionic, diamond, beta-decay, irradiation, semiconductor

\section{INTRODUCTION}

Thermionic emission is the thermally induced emission of electrons from the surface of a material. If the bulk temperature increases above a certain threshold, its electrons will have enough energy to surmount the surface potential barrier and escape from the solid with a certain kinetic and potential energy.

Thermionic emission has been used in a number of applications including vacuum diodes, electron guns, and X-ray sources. Another potential application of thermionic emission is in thermionic energy converters (TECs), which can produce a direct conversion of heat into electrical power using no moving parts (Gryaznov et al., 1989; Mills and Dahlberg, 1991).

One of the drawbacks of thermionic emission-based devices is their high operational temperature, which has so far restricted the application of thermionic emission to specialized fields like space exploration and scientific instrumentation. Nevertheless, with the advent of novel materials and newly discovered material properties, research on how to achieve thermionic emission at low temperatures has experienced a renewed interest. One such material is hydrogen terminated, nitrogen-doped nanocrystalline diamond (HTND). Both nitrogen incorporation and high grain boundary density are believed to enhance thermionic emission owing to enhancement of conductivity at elevated temperatures, with nitrogen also lowering the material work function (Wang et al., 2003). Upward 
band-bending at the vacuum interface contributes to an energy barrier to emission (Ibach, 2006). Electrons may either tunnel through this barrier or, given enough energy input, go over it. HTND has a property termed negative electron affinity (NEA), its conduction band minimum being above the vacuum electron energy. This property somewhat counteracts the barrier due to band bending, enabling conduction band electrons within the material to be emitted into the vacuum. The NEA surface can be considered a requirement for thermionic emission, since only $\mathrm{H}$ terminated diamond has consistently been shown to produce a thermionic current (Koeck et al., 2004, 2009; Paxton et al., 2012). Extensive studies on HTND give it potential as a candidate for the implementation of thermionic emission devices even at temperatures below $1,000 \mathrm{~K}$, where both material stability and efficient device operation are possible.

The theory by which thermionic emission is described is the so-called Richardson-Dushman model (Dushman, 1930), which is parameterized by the following:

$$
J=A T^{2} e^{-W / k T},
$$

where $J$ is the emission current density, $T$ is the surface temperature, $W$ is the surface work function, $k$ is Boltzman's constant, and $A$ is a factor derived from various material properties, and is poorly characterized for most materials (Modinos, 2013). This factor, combined with the assumption that surface chemistry is unchanged by temperature, renders the Richardson equation inadequate for a rigorous description of the emission from all but the most robust metals. That said, the model may be used for illustrative purposes, since emission current often follows an exponential-like trend with respect to temperature. A thermionic parameter used by previous authors in the evaluation of diamond emitters is the emission threshold temperature $T_{\mathrm{t}}$ (Paxton et al., 2012). Assessment of $T_{\mathrm{t}}$ not only mitigates the negative effects of temperature on the data (since it is the lowest temperature at which emission is detected) but provides a simpler metric from which to discern variation across an experiment than fitting to a somewhat unsatisfactory model. A combination of $T_{\mathrm{t}}$ with output current provides a reliable metric for the success of thermionic emission experiments.

So far, the results on thermionic emission from diamond films have been promising, making use of n-type diamond using nitrogen (Koeck et al., 2004) as well as phosphorus (Koeck et al., 2009) as primary dopants. However, due to their donor activation energies and induced structural distortions in the diamond lattice, these dopants only promote poor electrical conductivity, limiting the thermionic emission performance of HTND. With no satisfactory shallow donor available for diamond (Goss et al., 2008), the material suffers from stagnation with respect to progress toward technological maturity. The need arises for a method of increasing the low temperature thermionic performance of diamond thin films emitters.

Electromagnetic radiation has previously been employed to enhance the electronic emissivity of thermionic surfaces (Schwede et al., 2010; Sodha et al., 2017), where the electron emission from a hot p-type semiconductor was greatly increased when illuminated with light. This enhancement did not come from photoelectron emission as the effects could be seen when using photon energies below the work function, and instead was attributed to a boost of electron energies into a "quasi-Fermi level." This process involves the continuous photoexcitation of valence electrons into the conduction band, and the thermalization of such electrons within it. This generates a population of thermally distributed electrons above the conduction band minimum, increasing thermionic efficiency as a result.

As an alternative, this paper reports the use of beta radiation as a way to enhance thermionic emission from diamond. Beta particles are electrons of high speed and energies of tens to thousands of $\mathrm{keV}$ produced by the radioactive decay of unstable nuclei. Several diamond films were tested for thermionic emission with and without exposure to beta radiation from the decay of a ${ }^{63} \mathrm{Ni}$ beta source. The results presented demonstrate that beta radiation exposure enhances the thermionic emission properties of diamond. Finally, various mechanisms that could explain the observed improvement are discussed.

\section{MATERIALS AND METHODS}

\section{Fabrication of Emitter Structure}

Nitrogen-doped nanocrystalline diamond films were grown on $10 \mathrm{~mm} \times 10 \mathrm{~mm} \times 0.5 \mathrm{~mm}$ Mo substrates (Goodfellow, UK). Molybdenum is a suitable substrate owing to its high melting point, high thermal conductivity, strong carbide, and low coefficient of thermal expansion, which minimizes the mechanical stress on the diamond film. The molybdenum was seeded by submerging the substrates first in a carboxyethyl silanetriol di-sodium solution ( $25 \%$ in water, Fluorochem) and then a suspension of sonicated $18 \mathrm{~nm}$ nano-diamond particles $(25 \mathrm{cts} /$ $\mathrm{kg}$, Microdiamond AG) to aid nucleation.

Diamond growth was performed by microwave plasmaenhanced chemical vapor deposition (MWCVD) in a 1.5 kW ASTeX type reactor. During MWCVD growth, $2.45 \mathrm{GHz}$ frequency radiation is introduced into a $1 \mathrm{~L}$ volume cylindrical vacuum chamber through a quartz window at the top. The chamber forms a resonant cavity, with the antinode of the resulting mode producing a stationary plasma centrally above the substrate position. Heated entirely by the plasma, the substrate temperature is monitored by a single color optical pyrometer (Thermalert SX, $2.2 \mu \mathrm{m}$, Raytek, UK). The process gas mixture comprised $4 \%$ methane diluted in hydrogen, with an additional $0.4 \%$ nitrogen, giving a $\mathrm{CH}_{4}: \mathrm{N}_{2}$ ratio of $10: 1$, and a total flow rate of $315 \mathrm{sccm}$. The pressure was maintained at 130 Torr and the power at $1.3 \mathrm{~kW}$, resulting in a substrate temperature between 850 and $900{ }^{\circ} \mathrm{C}$. Films were grown for $15 \mathrm{~min}$, yielding a consistent film thickness of under $1 \mu \mathrm{m}$. The sample set used for this study consisted of eight films grown on identical substrates at identical input process conditions.

Each sample was analyzed using Raman spectroscopy (2000, Renishaw, UK) to confirm that all the HTND films were of similar composition. Measurements were taken with an excitation wavelength of $514 \mathrm{~nm}$. Additionally, the morphology of the surface was characterized with a field emission gun scanning electron microscope (Zeiss Sigma HD). 
Samples were hydrogen terminated by exposure to hydrogen plasma to induce a NEA. The MWCVD reactor allows facile and consistent (re-)termination using a 3-step process. The sample is first treated by 85 Torr of hydrogen with 1,200 W input power for 2 min to strip the surface of contaminants and chemisorbed species. This is followed by $2 \mathrm{~min}$ at 30 Torr with $700 \mathrm{~W}$ input power to allow hydrogen to chemisorb on vacant surface sites, producing the NEA-inducing dipole. A final cooling phase for 2 min in 30 Torr of hydrogen gas ensured no other gases could occupy any remaining vacant surface sites.

\section{Collector Structure}

The collector electrode was made from molybdenum foil, which had been perforated to allow beta radiation to pass through it from an embedded nickel-63 source (Ritverc GmbH, Germany). Nickel-63 has a $99 \%$ beta radiopurity, with a $17.4 \mathrm{keV}$ average and $70 \mathrm{keV}$ maximum particle energy. The preformed molybdenum cap held a $3 \mathrm{~mm} \times 3 \mathrm{~mm} \times 0.1 \mathrm{~mm}$ foil of either ${ }^{63} \mathrm{Ni}(2.6 \mathrm{MBq})$ or ${ }^{59} \mathrm{Ni}$ (zero activity control) and a $0.1 \mathrm{~mm}$ Mo spacer (Figure 1A). The perforated holes in the cap plate exposed $50.3 \%$ of the $\mathrm{Ni}$ foil, so that the sample was exposed to a total calculated activity of $1.3 \mathrm{MBq}$ using the Ni beta source.

\section{Laser-Heated Thermionic Emission Measurements}

The TEC diode housing (Figure 1B) could be removed to exchange both the embedded $\mathrm{Ni}$ foil and the emitter sample. When replaced, the inter-electrode gap was controlled by a linear motor (Z825V, Thorlabs, Germany) to ensure a constant spacing of $200 \mu \mathrm{m}$. A $25 \mathrm{~V}$ bias between the electrodes ensured that emitted electrons contributed to the measured current, while producing no measurable field emission.

The emitter assembly was encased on a stainless steel chamber (Figure 1C) and held under vacuum with a base pressure of $10^{-6}$ Torr. The back (non-diamond) surface of the sample is heated by a polarized $40 \mathrm{~W} \mathrm{CO}_{2}$ laser (Firestar V series, Synrad, UK) with a wavelength $10.6 \mu \mathrm{m}$ and spot-size of $\approx 6 \mathrm{~mm}$. A 2-color optical pyrometer (Spotmeter R160, Land-Ametek, UK, $<2.5 \mathrm{~K}$ uncertainty) monitored the sample temperature through a borosilicate window.

Automated temperature profiles were programmed to either periodically cycle the emitter samples between 300 and $600{ }^{\circ} \mathrm{C}$ (sample set A) at a rate of $5 \mathrm{~K} \mathrm{~s}^{-1}$, or ramp once from 300 to $800{ }^{\circ} \mathrm{C}$ ( sample set $\mathrm{B}$ ). This range was chosen because the HTND emission threshold was established to lie between 400 and $600^{\circ} \mathrm{C}$

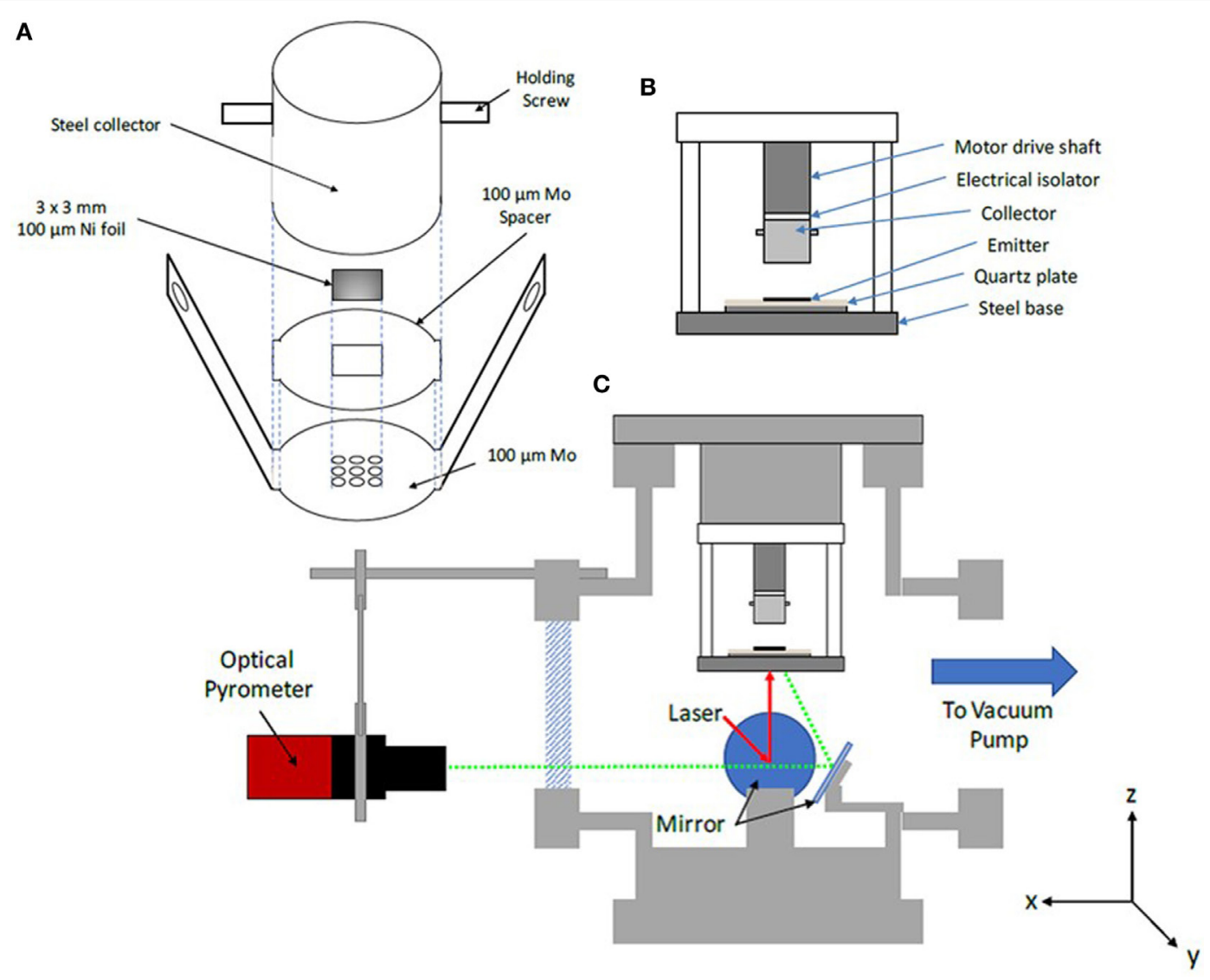

FIGURE 1 | Schematic representations of the experimental equipment. (A) show the molybdenum housing for the Ni foil within the collector, (B) shows each of the parts of the thermionic diode structure with electrical connections omitted for clarity, and (C) shows the entire test set-up, showing how both the laser ( $y$-axis) and pyrometer ( $x$-axis) are directed at the sample via mirrors. 
and temperatures higher than $600{ }^{\circ} \mathrm{C}$ cause rapid hydrogen desorption, which reduces the thermionic emission properties of diamond during cycling. The pyrometer, while directed at the back surface of the emitter structure, measured a consistent overshoot of $30{ }^{\circ} \mathrm{C}$ beyond the $600{ }^{\circ} \mathrm{C}$ set point in each cycle in sample set A.

\section{RESULTS}

\section{Material Characterization}

Raman spectra and scanning electron micrographs showed a satisfactorily consistent sample set. The characteristic diamond peak at $1,332 \mathrm{~cm}^{-1}$ was present in all samples, but, as shown in Figure 2A, ubiquitously appeared at $1,337 \mathrm{~cm}^{-1}$, indicative of the strain caused during cooling of the molybdenum substrate from growth- to room-temperature. Broad peaks appeared at $1,470-1,545$ and $1,140 \mathrm{~cm}^{-1}$, characteristic of graphitic $\mathrm{sp}^{2}$, and
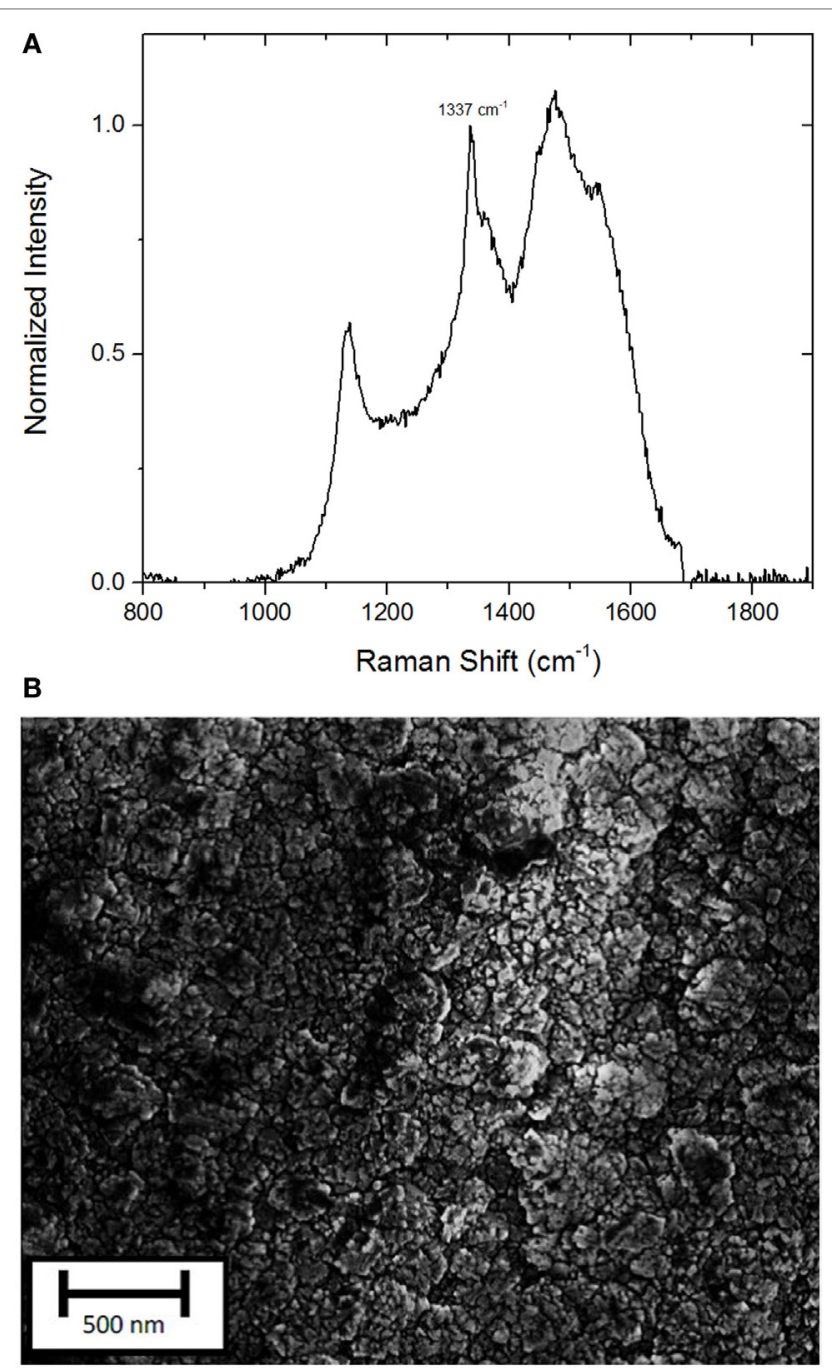

FIGURE 2 | Representative material characterization data for the diamond emitter surface. Both Raman spectra (A) and scanning electron micrographs (B) were consistent across the sample set. polymeric $\mathrm{sp}^{2}$ carbon, respectively, both of which are commonly seen in HTND Raman spectra (Kuzmany et al., 2004).

A representative SEM image of one of the diamond films used in this study is shown in Figure 2B, where the sub-100 nm grain size can be clearly identified. Qualitative similarity was found on all samples, with only minor variations in morphology and grain size due to slight temperature inconsistencies during growth.

\section{Thermionic Emission Experiment}

The thermionic emission characteristics of nominally identical samples demonstrate enough variability that it is not meaningful to compare one irradiated sample with another control sample. Statistical significance was attained by a sufficiently large sample set with multiple identically designed repeats on each sample. The experiment was thus carried out as follows.

A set of eight HTND samples was used to test thermionic emission under beta irradiation. Each sample was tested four times, twice under beta irradiation from ${ }^{63} \mathrm{Ni}$ (referred to as "beta"), and twice under no irradiation ("non-beta"). The order of beta and non-beta test runs was balanced such that any resulting bias was eliminated. Sample set A was examined by repeated cycling of the cathode temperature and showed the magnitude and stability of emission current both with and without beta irradiation. Whereas, sample set B was used in a direct comparison of the emission threshold temperature.

Figure 3 depicts the typical thermionic behavior of the diamond emitters during a single temperature cycle from 300 to $600{ }^{\circ} \mathrm{C}$. With the emission current density proportional (Eq. 1) to both the square and the exponential of the temperature, the drop in current due to the slight $\left(\sim 20^{\circ} \mathrm{C}\right)$ reduction in $\mathrm{T}$ after the overshoot is strongly pronounced in the current profile. This current drop is exaggerated by surface hydrogen desorption, which is hastened at higher temperatures. Desorption of the NEA-inducing dipole will effectively reduce the emitter area, hampering the ability of the cathode to produce current.

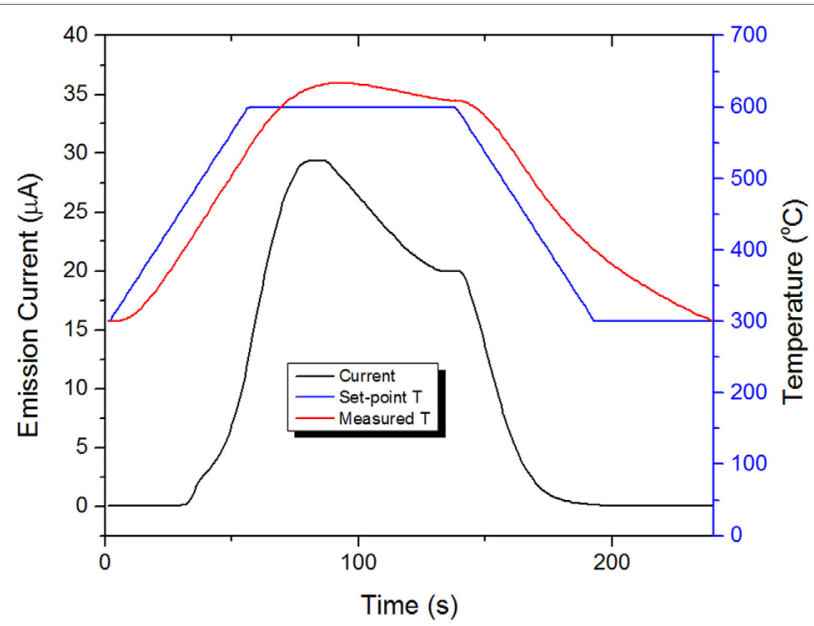

FIGURE 3 | An example temporal temperature cycle and emission current profile. This cycle is repeated 20 times for sample set A. Data were taken between 300 and $600{ }^{\circ} \mathrm{C}$ set-point temperature. The behavior at higher set-point temperatures is qualitatively similar to that shown here. 
This instability of the hydrogen surface results in the maximum emission current occurring $5{ }^{\circ} \mathrm{C}$ below the temperature maximum, as shown in Figure 3.

\section{Temporal Characteristics}

Figure 4A shows a plot of thermionic emission current with respect to temperature for consecutive temperature ramp cycles of a non-beta test run in the threshold region. If the thermionic emission data is fitted to the Richardson-Dushman model, then a work function of $\sim 2.2 \mathrm{eV}$ can be derived for each peak in the 20 -cycle temporal experiment. This value increased by $0.2 \mathrm{eV}$ over the full duration of the experiment, but the shape of the plots remains unaltered. Like previous authors (Paxton et al., 2012), we conclude that since hydrogen plasma treatment regenerates the emission characteristics between runs, this degradation is due to hydrogen desorption processes occurring rapidly at high temperatures.

The threshold emission temperature for the 20 cycles of the same sample is shown in Figure 4B. $T_{\mathrm{t}}$ is defined as the temperature at which the current surpasses $0.1 \mu \mathrm{A}$. The threshold

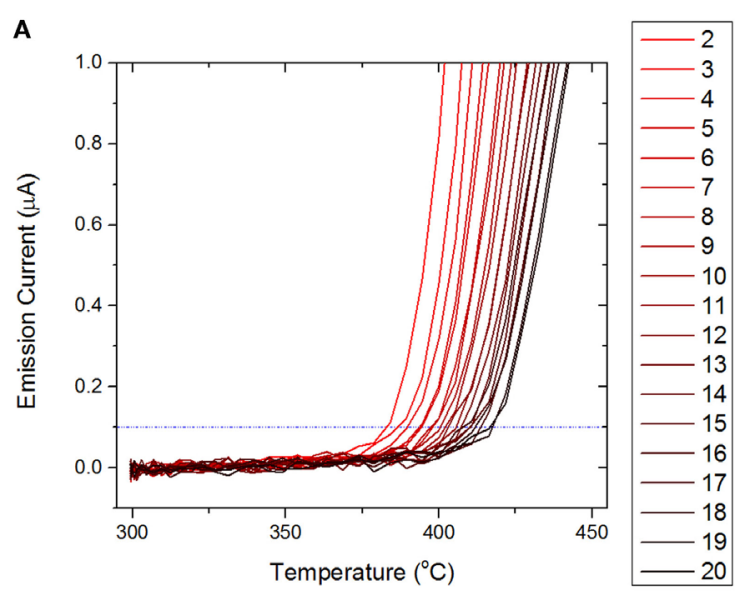

B

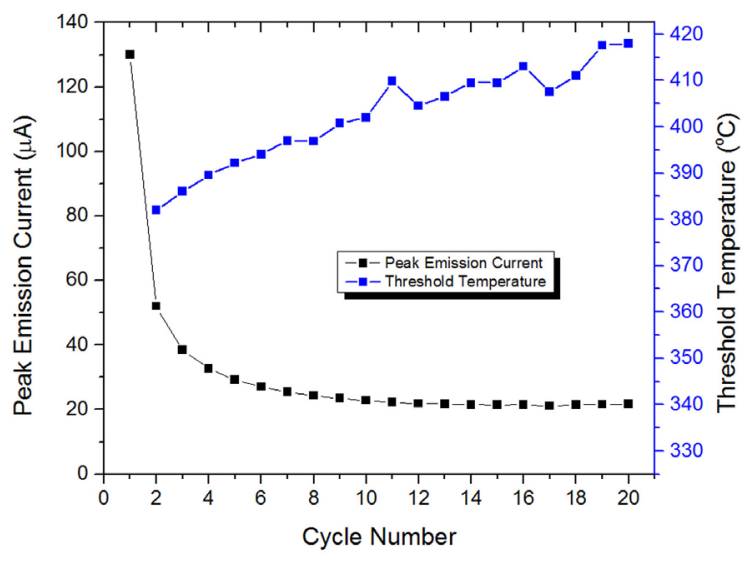

FIGURE 4 | Example characteristics for emission current as a function of temperature (A) and the peak emission current and threshold temperature as a function of cycle number (B), from a sample in set $\mathrm{A}$. $T$ was cycled between 300 and $600{ }^{\circ} \mathrm{C}$ over a period of $40 \mathrm{~min}$. Threshold temperature is defined where the current increases past $0.1 \mu \mathrm{A}$, shown by the blue dashed line. temperature increases by over $25^{\circ} \mathrm{C}$ when comparing the first and the last cycle. Alongside this, there is a striking decline in the current over the first five cycles, followed by a flattening at $\sim 15$ $\%$ of the initial peak current. This shows that lower threshold temperatures will ubiquitously provide a higher output current from diamond emitters provided surface chemistry remains constant. At the $600{ }^{\circ} \mathrm{C}$ temperature used in this experiment, not all of the hydrogen appears to be desorbed, allowing continued cycling of the experiment and resulting in a larger set of data for comparison of beta-exposed and non-exposed thermionic tests.

Sample set A was tested for damage by Raman spectroscopy after exposure to the beta radiation necessary to carry out this experiment. The spectra were unchanged by irradiation, with the new data reproducing the set of which Figure $2 \mathbf{B}$ is characteristic.

\section{Beta-Enhanced Thermionic Emission}

Figure 5 shows the average peak emission current of all the beta and non-beta tests in sample set $\mathrm{A}$. The $1.3 \mathrm{MBq}$ exposure induced on average a ratio of beta enhanced to non-beta enhanced current, or "enhancement factor," of 2.7 between the 2 nd and 20th cycles. Regarded unrepresentative, the first measurement cycle is ignored, an omission considered valid due to peak current and peak temperature being off-set far more than the average. This is likely due to the least stable hydrogen surface species being easily desorbed even at the modest temperatures used here. Enhancement is, however, still present in the first cycle current measurement.

Sample set B was tested purely as a measure of the enhancement effect of beta irradiation on the emission threshold temperature $T_{\mathrm{t}}$. Figure 6 shows an example of the data in the region close to the threshold. Of those examined, all samples showed lower $T_{t}$ values when exposed to beta radiation than without exposure. The average threshold temperature $T_{\mathrm{t} \text {,av }}$ of all the non-beta runs was $566 \pm 32{ }^{\circ} \mathrm{C}$, whereas that of the beta runs was $504 \pm 22^{\circ} \mathrm{C}$,

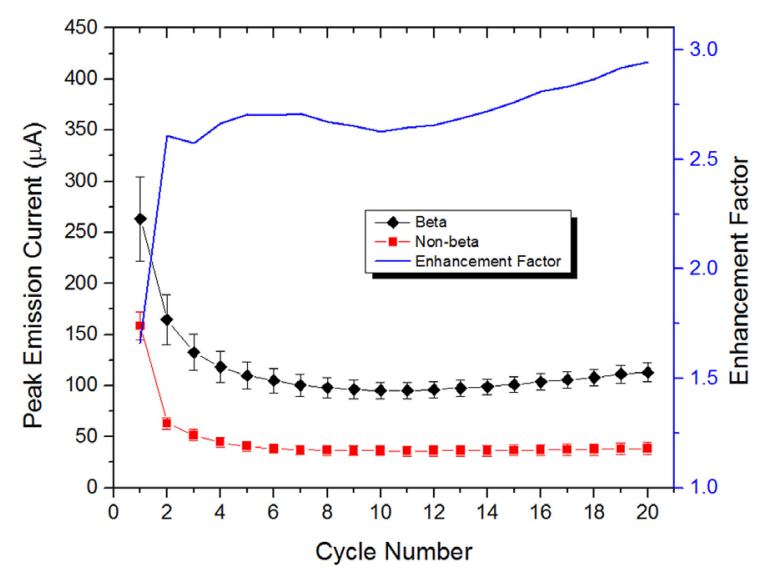

FIGURE 5 | Average temporal peak emission current values for sample set A, showing the beta ( $\left.{ }^{63} \mathrm{Ni}\right)$ current to be consistently higher than the non-beta $\left({ }^{5} \mathrm{Ni}\right)$ current. The enhancement factor (defined in the main text) is also shown on the right-hand axis. The error bars represent the standard error on the mean of all runs in sample set $A$. 


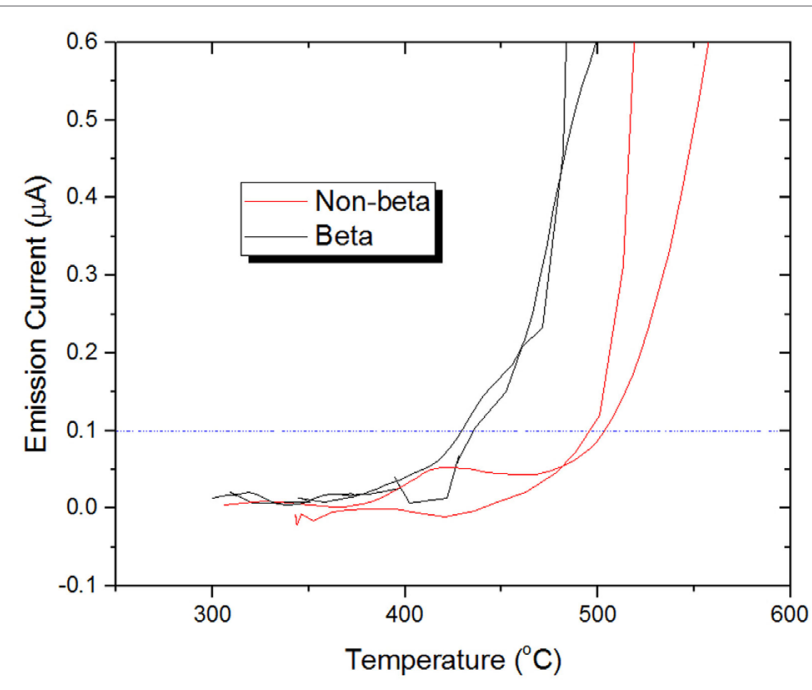

FIGURE 6 | Example characteristic emission current as a function of temperature in the threshold region for a sample in set B, with (black line) and without (red line) beta radiation exposure. The blue dashed line shows the current threshold considered to be thermionic emission.

where the quoted errors are standard errors on the mean of sample set $\mathrm{B}$. When the threshold temperature change of each sample is considered individually, the threshold temperature change average $\left(\Delta T_{\mathrm{t}}\right)_{\text {av }}$ is a clear reduction of $58 \pm 11^{\circ} \mathrm{C}$ from non-beta to beta in sample set $\mathrm{B}$.

\section{DISCUSSION}

There are several possible mechanisms that could explain the enhancement effect of thermionic current and threshold temperature produced by the beta radiation. Below, we examine them in detail in an effort to discern the most likely cause.

As the ${ }^{63} \mathrm{Ni}$ is emitting beta particles, it is possible they could be harvested in the collector through its positive bias. However, the background current measured in the collector below the threshold temperature is identical with and without the beta source and is on the order of $100 \mathrm{nA}$. This is, moreover, invariant under changes in anode bias. No enhancement of the current can, therefore, be caused by current generation in the collector anode, since this would happen even during times of no emission from the cathode.

It may be reasonable to expect the enhancement factor experienced in the emission to be caused by secondary electron emission (SEE). The beta particles could, upon contact with the surface and subsurface electrons, transfer sufficient energy to allow emission from the surface and capture by the biased collector. While SEE will most likely occur, it is trivial to show that no enhancement of this size is possible by secondary emission alone. With a nominal $50.3 \%$ of the beta particles reaching the emitting diamond surface and assuming $100 \%$ successful entrance into the material and interaction with charge carriers within it, then there are just over 13 million primary electrons per second. Given a generous secondary electron yield (secondary:primary ratio) of
100 (Dvorkin et al., 2003), the current returned would be on the order of 20 pA: a result far lower than that obtained in the data.

With a deep donor level and consequent low carrier concentration, nitrogen-doped diamond has a low room temperature electrical conductivity (Mort et al., 1991). As high-energy beta particles enter and travel through the diamond lattice, they generate electron-hole pairs. These extra charge carriers could increase the electrical conductivity of the diamond, in turn, increasing charge carrier replenishment at the surface. This mechanism could be responsible for the increase of thermionic current obtained herein and would be applicable in the case of nitrogendoped diamond, as it has a low carrier concentration. In order to ascertain the validity of this hypothesis, a new sample was grown on an insulating diamond substrate under conditions identical to those of sample sets A and B. In the same vacuum apparatus used for thermionic tests, the resistance of a diamond thin film was measured at both 300 and $600{ }^{\circ} \mathrm{C}$, with and without beta irradiation. This test produced no change in resistance within the precision of the experiment on the sample studied. To support this experimental evidence, we make a calculation based on the properties of electron-hole pairs found by previous researchers: with a generous (room temperature) exciton lifetime of $350 \mathrm{~ns}$ (Morimoto et al., 2016), and an energy of production of $13.3 \mathrm{eV}$ (Dimitrov et al., 2010), fewer than 600 electron-hole pairs will be activated at any given time within the diamond material.

The hydrogen termination may be considered a possible factor in the emission changes observed in the data. There are two possibilities regarding this hypothesis: First, chemically bonded $\mathrm{H}$ species on the surface could be desorbed by the high energy incoming radiation or in fact by outgoing secondary electrons. And second, any desorbed $\mathrm{H}$ atoms could become ionized, by beta or secondary, and add an ionic component to the existing electronic current. Both of these conceivable options are effects that would exist in the case of beta irradiation, but not in the non-beta case. Addressing the first point, it has been stated here and by others (Paxton et al., 2012) that the $\mathrm{H}$ termination of HTND is necessary for its thermionic emission properties. Thus, the assumption that as $\mathrm{H}$ desorbs from the surface the current will decrease, as is apparent at high temperatures, negates this enhancement mechanism. The second point is improbable, since electron impact ionization (EII) of $\mathrm{H}$ atoms is most likely to result in $\mathrm{H}+$ ions, which would act to lower the current generated in the system. H- ion product may be a small component of the EII; however, its effect on the current will be far outweighed by that of the $\mathrm{H}+$. Production of $\mathrm{H}-$ from secondary electrons would cause no change in the current, since emitted secondary electrons will contribute to current whether bound or not to a $\mathrm{H}$ atom. We, therefore, conclude that $\mathrm{H}$ termination is not playing a role in the thermionic current enhancement presented here.

Alteration of bulk material by high energy electrons, also known as radiation damage, has been reported for diamond materials (Campbell et al., 2002). Beta-induced defects may, therefore, be produced and could play a role in thermionic current enhancement. To do this, a defect would have to either (1) increase conductivity at thermionic temperatures, (2) increase conduction band population at thermionic temperatures, or (3) lower the work function, or otherwise facilitate emission from 
the surface. Vacancies and interstitials are produced in diamond by beta radiation of considerably higher energy than is released during the decay of ${ }^{63} \mathrm{Ni}$ (Koike et al., 1992; Campbell et al., 2002). In HTND, however, it is possible that nitrogen-vacancy centers may be produced at much lower energies (Schwartz et al., 2012). Nevertheless, none of these point defects are believed to increase conductivity in nominally n-type material, nor produce a donor type defect. Lowering the work function would require either surface dipole strengthening or some anisotropic subsurface defect that lowers the work function. Since the major surface disruption known for HTND is $\mathrm{H}$ desorption, interaction with $\mathrm{H}$ is unlikely to produce a stronger dipole. Furthermore, subsurface defects are predicted, at least for substitutional $\mathrm{N}$, to reduce the $\mathrm{H}$ bond strength (Yiming et al., 2014). Irradiation damage is, therefore, likely to result in a lower emission current in HTND.

As beta particles interact with the pre-existing valence band and conduction band electrons within the diamond, they will undergo inelastic energy transfer, removing the native electrons from equilibrium. The non-equilibrium electrons, also called hot electrons by other authors (Brongersma et al., 2015), will cause a distortion of the Fermi-Dirac (FD) distribution of electrons such that energy states with high energy will be populated. The barrier to emission due to upward band bending at the surface results in a proportion of conduction band electrons, which in a NEA material are considered to emit easily, being trapped within the bulk. When given a non-zero energy input, some number of these electrons could be promoted to a level above the barrier height. Skewing the conduction band electron distribution in this way could add a component to the emission-energy electron population and would result in an enhanced thermionic current, without significantly increasing conductivity. This is in contrast to the work of Schwede et al. (2010), where photon radiation enhanced current by increasing the total number of conduction band electrons.

The above hypothesis concurs with the findings presented in a study by Domenech-Garret et al. (2013) who examined the effect of non-equilibrium electrons on thermionic emission in metals. The researchers reformulated the FD statistics for a system of excited electrons by approximating the energy spectrum using a Kappa term in the energy distribution function. This so-called Kappa distribution, common in Plasma Physics and Astrophysics (Livadiotis and McComas, 2013), is applied to thermionic emission current. The exponent in the FD distribution is not an exponent in the work of Domenech-Garret et al., but is itself raised to the power of the parameter Kappa, the value of which is said to be dependent on the non-equilibrium nature of the distribution. The shape of the FD distribution is recovered at high values of Kappa, but at low values with a large distortion the thermionic emission

\section{REFERENCES}

Brongersma, M. L., Halas, N. J., and Nordlander, P. (2015). Plasmon-induced hot carrier science and technology. Nat. Nanotechnol. 10, 25-34. doi:10.1038/ nnano.2014.311

Campbell, B., Choudhury, W., Mainwood, A., Newton, M., and Davies, G. (2002). Lattice damage caused by the irradiation of diamond. Nucl. Instrum. Methods Phys. Res. A 476, 680-685. doi:10.1016/S0168-9002(01)01664-3 current increases by multiple orders of magnitude. This implies that non-thermal alteration of the electron energy distribution may provide a means to increase thermionic output considerably. Whether this Kappa distribution is the key to understanding the interaction of beta radiation with the enhanced thermionic current is unclear, and we leave this question open for further research.

\section{CONCLUSION}

In summary, this report shows that exposure to beta radiation enhances the output of HTND thermionic emitters. When irradiated with high energy beta particles, both the peak thermionic emission current at $600{ }^{\circ} \mathrm{C}$ and the emission threshold temperature are altered. Peak current is enhanced by a factor of 2.7 on average, and threshold temperature is reduced by $58 \pm 11^{\circ} \mathrm{C}$, when compared to a non-irradiated control. Various mechanisms that could explain the beneficent effects are discussed, with most deemed unlikely or ineffectual. One possible explanation, involving a skewing of the excited state distribution of electron density toward higher energy states, appears convincing, and is potentially associated with a Kappa parameter distribution. Nevertheless, the mechanism of enhancement is an open problem. Regardless of the mechanism, we consider beta-enhancement to be a promising step toward realization of HTND thermionic emission technologies.

\section{AUTHOR CONTRIBUTIONS}

AC, GW, AR generated all the experimental data and the draft manuscript. JS assisted with experimental setup. HA designed the thermionic test rig and assisted with the draft manuscript, NF advised on experimental design and assisted with the preparation of the manuscript.

\section{ACKNOWLEDGMENTS}

We thank John Rowden and Adrian Crimp (Bristol Physics Mechanical and Glass Workshops) for their assistance with the design and construction of the laser-heated test rig. We also thank Dr Sara Nunez-Sanchez and Prof. Martin Cryan (Department of Electrical and Electronic Engineering, University of Bristol) for their help and advice with the design of the laser-machined Molybdenum grating absorber.

\section{FUNDING}

This work was supported by the Engineering and Physical Sciences Research Council under grant no. EP/K030302/1/46623. diamond. J. Appl. Phys. 108, 073712. doi:10.1063/1.3491041

Domenech-Garret, J. L., Tierno, S. P., and Conde, L. (2013). Enhanced thermionic currents by non equilibrium electron populations of metals. Eur. Phys. J. B 86, 382-385. doi:10.1140/epjb/e2013-40646-5

Dushman, S. (1930). Thermionic emission. Rev. Mod. Phys. 2, 381-476. doi:10.1103/ RevModPhys.2.381 
Dvorkin, V. V., Dzbanovsky, N. N., Suetin, N. V., Poltoratsky, E. A., Rychkov, G. S., Il'Ichev, E. A., et al. (2003). Secondary electron emission from CVD diamond films. Diamond Relat. Mater. 12, 2208-2218. doi:10.1016/S0925-9635 (03)00320-0

Goss, J. P., Eyre, R. J., and Briddon, P. R. (2008). Theoretical models for doping diamond for semiconductor applications. Phys. Status Solidi B Basic Res. 245, 1679-1700. doi:10.1002/pssb.200744115

Gryaznov, G. M., Zhabotinskii, E. E., Zrodnikov, A. V., Nikolaev, Y. V., Pupko, V. Y., Serbin, V. I., et al. (1989). Thermoemission reactor-converters for nuclear power units in outer space. Soviet Atom. Energy 66, 414-418. doi:10.1007/BF01123508

Ibach, H. (2006). Physics of Surfaces and Interfaces, Vol. 12. Berlin: Springer.

Koeck, F. A. M., Garguilo, J. M., and Nemanich, R. J. (2004). On the thermionic emission from nitrogen-doped diamond films with respect to energy conversion. Diamond Relat. Mater. 13, 2052-2055. doi:10.1016/j.diamond.2004. 06.027

Koeck, F. A. M., Nemanich, R. J., Lazea, A., and Haenen, K. (2009). Thermionic electron emission from low work-function phosphorus doped diamond films. Diamond Relat. Mater. 18, 789-791. doi:10.1016/j.diamond.2009.01.024

Koike, J., Parkin, D. M., and Mitchell, T. E. (1992). Displacement threshold energy for type IIa diamond. Appl. Phys. Lett. 60, 1450-1452. doi:10.1063/1.107267

Kuzmany, H., Pfeiffer, R., Salk, N., and Günther, B. (2004). The mystery of the 1140 cm-1 Raman line in nanocrystalline diamond films. Carbon N. Y. 42, 911-917. doi:10.1016/j.carbon.2003.12.045

Livadiotis, G., and McComas, D. J. (2013). Understanding kappa distributions: a toolbox for space science and astrophysics. Space Sci. Rev. 175, 183-214. doi:10.1007/s11214-013-9982-9

Mills, J. C., and Dahlberg, R. C. (1991). Thermionic systems for DOD missions. AIP Conf. Proc. 217, 1088-1092. doi:10.1063/1.40069

Modinos, A. (2013). Field, Thermionic and Secondary Electron Emission Spectroscopy. New York: Springer Science \& Business Media.

Morimoto, H., Hazama, Y., Tanaka, K., and Naka, N. (2016). Exciton lifetime and diffusion length in high-purity chemical-vapor-deposition diamond. Diamond Relat. Mater. 63, 47-50. doi:10.1016/j.diamond.2015.11.010
Mort, J., Machonkin, M. A., and Okumura, K. (1991). Compensation effects in nitrogen-doped diamond thin films. Appl. Phys. Lett. 59,3148-3150.doi:10.1063/ 1.105767

Paxton, W. F., Howell, M., Kang, W. P., and Davidson, J. L. (2012). Influence of hydrogen on the thermionic electron emission from nitrogen-incorporated polycrystalline diamond films. J. Vacuum Sci. Technol. B30, 021202. doi:10.1116/ 1.3684982

Schwartz, J., Aloni, S., Ogletree, D. F., and Schenkel, T. (2012). Effects of low-energy electron irradiation on formation of nitrogen-vacancy centers in single-crystal diamond. New J. Phys. 14, 043024. doi:10.1088/1367-2630/14/4/043024

Schwede, J. W., Bargatin, I., Riley, D. C., Hardin, B. E., Rosenthal, S. J., Sun, Y., et al. (2010). Photon-enhanced thermionic emission for solar concentrator systems. Nat. Mater. 9, 762-767. doi:10.1038/nmat2814

Sodha, M. S., Srivastava, S., and Mishra, R. (2017). Enhancement of thermionic emission by light. Eur. Phys. J. Appl. Phys. 77, 20101. doi:10.1051/epjap/ 2017160206

Wang, S. G., Zhang, Q., Yoon, S. F., Ahn, J., Zhou, Q., Wang, Q., et al. (2003). Electron field emission enhancement effects of nano-diamond films. Surf. Coat. Technol. 167, 143-147. doi:10.1016/S0257-8972(02)00901-5

Yiming, Z., Larsson, F., and Larsson, K. (2014). Effect of CVD diamond growth by doping with nitrogen. Theor. Chem. Acc. 133, 1-12. doi:10.1007/ s00214-013-1432-y

Conflict of Interest Statement: The authors declare that the research was conducted in the absence of any commercial or financial relationships that could be construed as a potential conflict of interest.

Copyright (c) 2017 Croot, Wan, Rowan, Andrade, Smith and Fox. This is an openaccess article distributed under the terms of the Creative Commons Attribution License (CC BY). The use, distribution or reproduction in other forums is permitted, provided the original author(s) or licensor are credited and that the original publication in this journal is cited, in accordance with accepted academic practice. No use, distribution or reproduction is permitted which does not comply with these terms. 